\title{
Reflexion and refraction coefficients (amplitudes) of plane elastic waves at the boundary between transversely isotropic media
}

\author{
1st Part
}

Gulzar AmMaD

Ricevuto il 15 Maggio 1967

Riassunto. - Con le equazioni che sono servite al calcolo dei rapporti delle ampiezze fra le onde riflesse e rifratte, e le onde quasi-longitudinali e quasi-trasversali incidenti su una superficie limite fra due mezzi trasversalmente isotropi, sono state ricavate anche le direzioni di polarizzazione delle onde stesse.

Si sono fatte, inoltre, alcune particolari interessanti applicazioni delle equazioni trovate. I rapporti delle ampiezze delle onde originate, sono stati calcolati per l'onda incidente quasi-longitudinale, quando gli assi di simmetria dei mezzi coincidono con la normale alla superficie limite.

Nei diagrammi riportati nella nota, si vede chiaramente come i rapporti delle ampiezze vengano influenzati dal variare dei diversi parametri elastici.

Summary. - The equations leading to the calculation of the amplitude ratios of the reflected and refracted waves to the incident quasilongitudinal and quasitransversal waves at a boundry surface between two transversely isotropic media have, been derived along with their polarisation directions. Some interesting specialisations of the equations have also been given. The amplitude ratios of the derived waves have been calculated for the incident quasilongitudinal wave, when the symmetry axes of the media coincide with the normal to the boundry surface. The influence of varying the different elastic parameters has been presented in the diagrams.

Zusammenfassung. - Die linearen Gleichungen, deren Loesung die Amplitudenverhaeltnisse der reflektierten und gebrochenen Wellen zur einfal. 
lenden quasilongitudinalen und quasitransversalen Welle an einer Grenzflaeche zwischen zwei transversalisotropen Medien ergeben, sind abgeleitet worden. Die Polarisationsrichtungen der Wellen sind auch angegeben. Die Gleichungen sind fuer einige Sonderfaelle spezialisiert worden. Die Amplitudenverhacltnisse sind fuer den Fall berechnet worden, in dem die Symmetriachsen beider Medien mit der Grenzflaechennormale zusammenfallen. Der Linfluss der Variation verschiedener elastischer Parameter auf die Amplituden ist in einigen Diagrammen dargestellt.

The reflexion and transmission coefficients for the plane elastic waves on the plane discontinuities between homogeneous isotropic media were derived by Knott (1) for the first time. Since then several authors e.g. Zoeppritz ( $\left.{ }^{2}\right)$, Muskat et al. $\left({ }^{3}\right)$, Koefoed (') have investigated the influence of the different elastic parameters on them. But as the methods of the interpretation of the applied seismics were improved, it was found that the assumption of isotropy for the media is in many cases doubtful and in certain definitely untenable. It has been shown several times that the uppermost layers of the Earth's crust are anisotrope over a few thousand meters e.g. see Uhrig et al. $\left(^{5}\right)$. The ultrasonic measurements of the elastic wave velocities in some rock samples have shown clearly their dependence on the direction of the measurement $\left({ }^{9}\right)\left({ }^{7}\right)\left({ }^{8}\right)\left({ }^{9}\right)$, has examined the implication of the anisotropy for the crustal and upper mantle structure. Recently Vvedenskaya et al. $\left({ }^{10}\right)$, Nuttli et al. (11) and Meissner (12) have tried to show the connexion of the recorded amplitudes and directions of polarisation of the $P$ - and $S$-waves with the possibility of the presence of anisotropy in the mantle and crust. The complicated behaviour of a few travel-time curves conld also be explained through the assumption of the anisotropy. The theoretical considerations have also led to the conclusion that the spacial inhomogeneities, which are definitely present, must give rise to the elastic aeolotropy, mostly transversal or hexagonal e.g. Postma $\left({ }^{13}\right)$, Backus $\left({ }^{14}\right)$. In order to take these facts into consideration, the influence of the anisotropy on the reflexion and transmission coefficients has been investigated for a few simple cases, for example Ossipor $\left({ }^{15}\right)\left({ }^{16}\right)$, Musgrave $\left({ }^{17}\right)$. In the present article these coefficients have been calculated for two transversalisotropic media in welded contact, which have a certain orientation to each other (see below). The explicite formulas for the arbitrary orientation has also been derived. 
The wave equations for the anisotropic media can be written down quite generally under the assumption of linear elasticity, no dispersion etc. as follows:

$$
C_{i j k l} \frac{\partial^{2} u_{k}}{\partial w_{j} \dot{\partial} \tilde{x}_{l}}=\varrho \cdot \frac{\partial^{2} u_{i}}{\partial t^{2}}+F_{i}
$$

where:

$\mathrm{C}_{\mathfrak{s} k l}=$ the components of the elastic tensor,

$\varrho=$ the density of the medium,

$\left(x_{i}\right) x_{k}=$ the orthogonal cartesian coordinates of a point of mass, $\left(u_{k}\right) u_{i}=$ the components of the displacement of the point in the direction $\left(x_{k}\right) x_{i}$.

$F_{i}$ - the component of the body force along the $x_{i}$-axis.

It has often been shown that there exist three different waves with different velocities in every direction and that the displacement vectors associated with them are orthogonal, and further that in the case of the transversalisotropy (hexagonal type) all waves perpendicular to a certain axis (mostly vertical in nature) are equivalent. For such a special case the wave equations become:

$$
\begin{aligned}
& \varrho \frac{\partial^{2} u}{\partial t^{2}}=A \frac{\partial^{2} u}{\partial x^{2}}+L \frac{\partial^{2} u}{\partial z^{2}}+(L+F) \frac{\partial^{2} w}{\partial x \partial z}+N \frac{\partial^{2} u}{\partial y^{2}}+(A-2 N) \frac{\partial^{2} v}{\partial x \partial y} \\
& \varrho \frac{\partial^{2} v}{\partial t^{2}}=A \frac{\partial^{2} v}{\partial y^{2}}+N \frac{\partial^{2} v}{\partial x^{2}}+L \frac{\partial^{2} v}{\partial z^{2}}+(A-2 N) \frac{\partial^{2} u}{\partial x \partial y}+L \frac{\partial^{2} v}{\partial z \partial y} \\
& o \frac{\partial^{2} v}{\partial t^{2}}=C \frac{\partial^{2} w}{\partial z^{2}}+L \frac{\partial^{2} v}{\partial x^{2}}+(L+F) \frac{\partial^{2} u}{\partial x \partial z}+(L+F) \frac{\partial^{2} v}{\partial y \partial z}+L \frac{\partial^{2} w}{\partial y^{2}}
\end{aligned}
$$

Here the notation introduced by Love $\left({ }^{18}\right)$ has been used, that is:

$$
\begin{array}{ll}
A=C_{11} & L=C_{44} \\
C=C_{33} & F=C_{13}
\end{array} \quad N=\left(\tilde{U}_{12}-C_{12}\right) / 2 .
$$

As all directions in $x-y$-plane are equivalent here, one should make the full use of this symmetry of rotation: for the plane wave the $y$-axis should be taken in the direction of the trace of the wave front on 
the $x$-y-plane. Thus one gets (neglecting the elements containing $\partial^{2} / \partial y^{2}$ and $\left.\partial / \partial y^{1}\right)$ :

$$
\begin{aligned}
& \varrho \frac{\partial^{2} u}{\partial t^{2}}=A \frac{\partial^{2} u}{\partial x^{2}}+L \frac{\partial^{2} u}{\partial z^{2}}+(L+F) \frac{\partial^{2} w}{\partial x \cdot \partial z} \\
& \varrho \frac{\partial^{2} v}{\partial t^{2}}=N \frac{\partial^{2} v}{\partial x^{2}}+L \frac{\partial^{2} v}{\partial z^{2}} \\
& \varrho \frac{\partial^{2} w}{\partial t^{2}}=L \frac{\partial^{2} w}{\partial x^{2}}+C \frac{\partial^{2} w}{\partial z^{2}}+(L+F) \frac{\partial^{2} u}{\partial z \cdot \partial x} .
\end{aligned}
$$

EXPRESSIONS FOR THE PAR'ICLE MOTION IN THE DIFFERENT WAVES

It can be seen from Eq. [3b] that it contains only $v$ and is independent of $u$ and $w$. That means that this wave is polarised in the direction perpendicular to that of the propagation and the displacement vector is parallel to the symmetry plane. An expression for such a plane wave can be written quite easily.

One sees from Eqs. [3b, 3c] that these waves are polarised in $x$-zplane. To derive the expression for them the plane wave may be written as follows:

$$
\begin{aligned}
& u=U \exp i \hbar\left(x \sin \varphi+z \cos \varphi-V_{n} t\right) \\
& w=W \exp i k\left(x \sin \varphi+z \cos \varphi-V_{n} t\right)
\end{aligned}
$$

where:

$$
\begin{aligned}
\varphi= & \text { the angle between the wave normal and the normal to } \\
& \text { the discontinuity, } \\
l= & \text { wave number }=2 \pi / \text { (wave length), } \\
U, W= & \text { the components of the displacement vector in the } x \text { - } \\
& \text { and } z \text {-axis, } \\
V_{n}- & V(\varphi)=\text { the phase velocity in the } \varphi \text {-direction. }
\end{aligned}
$$

If one puts Eq. [t] in Eqs. [3b, 3c], one gets:

$$
\begin{aligned}
& \left(A \sin ^{2} \varphi+L \cos ^{2} \varphi-\varrho V_{n}^{2}\right) U+(L+F) \sin \varphi \cos \varphi W=0 \\
& (L+F) \sin \varphi \cos \varphi U+\left(L \sin ^{2} \varphi+C \cos ^{2} \varphi-\varrho V_{n}^{2}\right) W=0
\end{aligned}
$$


Eq. [5] gives further [7]:

$$
\begin{aligned}
& \frac{U}{(L+F) \sin \varphi \cos \varphi}-\frac{W}{A \sin ^{2} \varphi+L \operatorname{co}^{2} \varphi-\varrho V^{2}(\varphi)}= \\
& =\frac{ \pm \sqrt{U^{2}+W^{2}}}{\sqrt{(L+F)^{2} \sin ^{2} \varphi \cos ^{2} \varphi+\left(A \sin ^{2} \varphi+L \cos ^{2} \varphi-\varrho V^{2}(\varphi)\right)^{2}}}= \\
& =\frac{ \pm G}{\sqrt{(L+F)^{2} \sin ^{2} \varphi \cos ^{2} \varphi+\left(A \sin ^{2} \varphi+L \cos ^{2} \varphi-\varrho V^{2}(\varphi)\right)^{2}}}
\end{aligned}
$$

with $G=\sqrt{U^{2}+W^{2}}=$ the total amplitude.

The signs of $G$ in [7] can be chosen according to the definition of the direction of the particle motion in the different waves.

For the non-trivial solution for $U$ and $W$, one must put the determinant of the coefficient matrix equal to zero and solve it. One gets thus the velocity as a function of the direction $\varphi$ :

$2 \varrho V_{1,2}^{2}(\varphi)=\left(\bar{A}-\bar{C} \sin ^{2} \varphi+L+C\right) \pm$

$\pm \sqrt{\left(\overline{A-C} \sin ^{2} \varphi+L+C\right)^{2}-4\left(\overline{A-L} \sin ^{2} \varphi+L\right)\left(L-C \sin ^{2} \varphi+C\right)+}$

$+4(L+F)^{2} \sin ^{2} \varphi \cos ^{2} \varphi$.

The plus sign belongs to the quasilongitudinal wave $(q L-)$, as this corresponds to the longitudinal wave, if the medium were isotrope. For a similar reason the negative sign belongs to the quasitransversal (qT-) wave.

The direction of the displacement vector as a function of wave normal can be determined as follows:

$$
\begin{gathered}
\cos \alpha: \cos \beta=\frac{U: W}{\sqrt{U^{2}+W^{2}}}= \\
=\frac{(L+F) \sin \varphi \cos \varphi:-\left(\overline{A-L} \sin ^{2} \varphi+L-\varrho V^{2}(\varphi)\right)^{2}}{\sqrt{(L+F)^{2} \sin ^{2} \varphi \cos ^{2} \varphi+\left(\overline{A-L} \sin ^{2} \varphi+L-\varrho V^{2}(\varphi)\right)^{2}}} .
\end{gathered}
$$

(Naturally one can use Eq. [6] instead of [5]. Both of the relations so obtained are equivalent due to Eq. [8]). 
Thus one gets the following expressions for the $q L$ - and $q T$-waves (the two solutions of $V$ in Eq. [8] are put into Eq. [10] and [11] and they determine the type of the wave):

$$
\begin{aligned}
u= & \pm \frac{G}{N(\varphi)} \cdot(L+F) \sin \varphi \cos \varphi \exp i k(x \sin \varphi+z \cos \varphi-V(\varphi) t) \\
w= \pm & \frac{G}{N^{r}(\varphi)}\left(A-L \sin ^{2} \varphi+L-\varrho V-(\varphi)\right) \\
& \quad \exp i k(x \sin \varphi+z \cos \varphi-V(\varphi) t)
\end{aligned}
$$

with:

$N^{2}(\varphi)=(L+F)^{2} \sin ^{2} \varphi \cos ^{2} \varphi+\left(\overline{A-L} \sin ^{2} \varphi+L-\varrho V^{2}(\varphi)\right)^{2} \cdot[11]$

As one sees, the different waves are described by the expressions of the same form, where one has only to assign the proper values of $V(\varphi)$ in order to get the required wave, from now onwards the $q L$ and $q T$-waves would be called the waves of the I and II type respectively. They would be assigned the same indices. The expressions in the medium II (one not containing the source) would be represented by astrisks e.g. $A^{*}, C^{*}, \ldots$ etc. etc.

From [10] and [11] one can easily show that their displacement vectors are orthogonal.

REFLEXION AND TRANSMISSION.

Whenever a wave strikes a surface of discontinuity it is partly reflected and partly refracted at different angles, which are determined through the Snell's law, that is here:

$$
\frac{\sin \alpha}{V(\alpha)}=\frac{\sin \beta}{V_{i}(\beta)}=-\frac{\sin \gamma}{V_{i}^{*}(\gamma)}
$$

where

$\alpha=$ the angle of incidence,

$\beta=$ the reflexion angle,

$\gamma=$ the angle of refraction,

$i=1,2$ according to the wave type,

$V_{1}(a)=$ the phase velocity, Eq. [8], in the direction $a$, measured from the normal to the surface of discontinuity. 
One can write the expressions of the components of the amplitudes for the different waves as follows:

(1) Incident wave of I or II type

$u=-\frac{G_{e}}{N(\alpha)} \cdot(L+F) \sin \alpha \cos \alpha \exp i \omega\left(\frac{x \sin \alpha+z \cos \alpha}{V_{1,2}}-t\right)$

$w=\frac{G_{e}}{N(\alpha)}\left(\overline{A-L} \sin ^{2} \alpha+L-\varrho V_{1,2}^{2}\right) \exp i \omega\left(\frac{x \sin \alpha+z \cos \alpha}{V_{1,2}}-t\right)$

(2) reflected wave of I type

$\left.u=-\frac{G_{r 1}}{N(\beta)} \cdot(L+F) \sin \beta \cos \beta \exp i \omega \frac{(x \sin \beta-z \cos \beta}{V_{1}}-t\right)$

$\left.w=-\frac{G_{r 1}}{N(\beta)}(A-L) \sin ^{2} \beta+L-\varrho V_{1}^{2}\right) \exp i \omega\left(\frac{x \sin \beta-z \cos \beta}{V_{1}}-t\right)$

(3) reflected wave of II type

$u=-\frac{G_{\tau^{2}}}{N(\gamma)}(L+F \sin \gamma \cos \gamma) \exp i \omega\left(\frac{x \sin \gamma-\cos \gamma}{V_{2}}-t\right)$

$w=-\frac{G_{\tau 2}}{N(\gamma)}\left(A-L \sin ^{2} \gamma+L-\varrho V_{2}^{2}\right) \exp i \omega\left(\frac{x \sin \gamma-z \cos \gamma}{V_{2}}-t\right)$

(4) refracted wave of I type

$u=-\frac{G_{d 1}}{N^{*}(\delta)}\left(L^{*}+F^{*}\right) \sin \hat{o} \cos \delta \exp i \omega\left(\frac{x \sin \delta+z \cos \delta}{V_{1}^{*}}-t\right)$

$w=+\frac{G_{d 2}}{N^{*}(\delta)}\left(\overline{A^{*}-L^{*}} \sin ^{2} \delta+L^{*}-\varrho^{*} V_{1}^{* 2}\right) \exp i \omega\left(\frac{x \sin \delta+z \cos \delta}{V_{1}^{*}}-t\right)$

(5) refracted wave of II type

$u=\frac{G_{d 2}}{N^{*}(\eta, 1}\left(L^{*}+F^{*}\right) \sin \eta \cos \eta \exp i \omega\left(\frac{x \sin n+z \cos n}{V_{2}^{*}}-t\right)$

$w=-\frac{G_{d 2}}{N^{*}(\eta)}\left(\overline{A^{*}-L^{*}} \sin ^{2} \eta+L^{*}-\varrho^{*} V_{2}^{* 2}\right) \exp i \omega\left(\frac{x \sin \eta+z \cos \eta}{V_{2}^{*}}-t\right)$.

Here $\nabla_{1,2}=V_{1}$ or $V_{2}$ according to the wave type.

(For the angles see Fig. 2).

The reflection and transmission coefficients have been defined in the literature for the isotropic media as follows:

(a) as the ratio of the amplitudes of the derived waves to that of the incident wave $\left({ }^{19}\right)\left({ }^{20}\right)$;

(b) as the ratios of the elastic potentials between the different waves and the incident wave $\left({ }^{21}\right)$. 
Since one cammot separate the elastic potentials associated with the different waves in the anisotropic media, the coefficients defined under $(b)$ do not have any meaning. Here they are defined therefore as in $(a)$.

\section{ARBITRARY ORIENTATION OF THE SYMMETRY AXES.}

A situation, which sometime occurs in nature, is that the media on both sides of the boundary surface separating them have their symmetry axes at some angle to the normal to the surface. One may think of, for example, the unconformities, where the horizontal strata are tilted, eroded and subsequently overlain by new sediments. In such a case one would find the symmetry axis in the underlying medium at the angle of tilting to the unconformity, whereas in the overlying medium it is perpendicular to it. Below the expressions for the reflexion and transmission coefficients are derived for a case, where the symmetry axes in both media are not perpendicular to the boundry. The above mentioned case would then be obtained by letting the axis coincide with the normal to the boundary.

Let the angle between the normal to the boundary and the symmetry axis in medium I (containing the source) and II be $\varphi$ and $\varphi^{*}$ respectively. The normal and the tangential components of the displacements and stresses can be obtained as follows: $u$ and $v$ are transformed as vectors and stresses as tensors of second order under the rotation of the axes:

$$
\begin{aligned}
& u=u^{\prime \prime} \cos \varphi-w^{\prime \prime} \sin \varphi \\
& w=u^{\prime \prime} \sin \varphi+w^{\prime \prime} \cos \varphi
\end{aligned}
$$

and

$$
\tau_{i j}=a_{i k \prime} a_{j l \prime} \tau_{k^{\prime \prime} l \prime},
$$

with $k^{\prime \prime}, l^{\prime \prime}, i$ and $j=1,2,3$ and $a_{i k^{\prime \prime}}$ as the cosine of the angle between $x_{i}$ and $x_{k^{\prime \prime-a x e s}}$ (see Fig. 1).

For the stresses one has:

$$
\begin{aligned}
& \tau_{23}-\tau_{y z}=\tau_{x^{\prime \prime} y^{\prime \prime}} \sin \varphi+\tau_{y^{\prime \prime} z^{\prime \prime}} \cos \varphi \\
& \tau_{13}-\tau_{x z}=\left(\tau_{x^{\prime \prime} x^{\prime \prime}}-\tau_{z^{\prime \prime} z^{\prime \prime}}\right) \sin \varphi \cos \varphi+\cos 2 \varphi \tau_{x^{\prime \prime} z^{\prime \prime}} \\
& \tau_{33}-\tau_{z z}=\tau_{x^{\prime \prime} x^{\prime \prime}} \sin ^{2} \varphi+\tau_{z^{\prime \prime}} \cos ^{\prime \prime} \varphi+\tau_{x^{\prime \prime} z^{\prime \prime}} \sin 2 \varphi .
\end{aligned}
$$


As mentioned above the pure transversal wave Eq. [3b] has its displacement only in the $y$-direction. According to Eq. [1t] and the definitions of $\tau_{z x}, \tau_{y z}$ etc. etc. it give rise to $\tau_{x y}, \tau_{z y}$ only, as generally:

$$
\begin{aligned}
& \tau_{z y}=L(\partial v / \partial z+\partial w / \partial y)=L \frac{\partial v}{\partial z} \\
& \tau_{z x}=L(\partial u / \partial z+\partial w / \partial x) \\
& \tau_{z z}=C \partial w / \partial z+F \partial u / \partial x \\
& \tau_{x y}=N(\partial u / \partial y+\partial v / \partial x)=N \frac{\partial v}{\partial x} .
\end{aligned}
$$

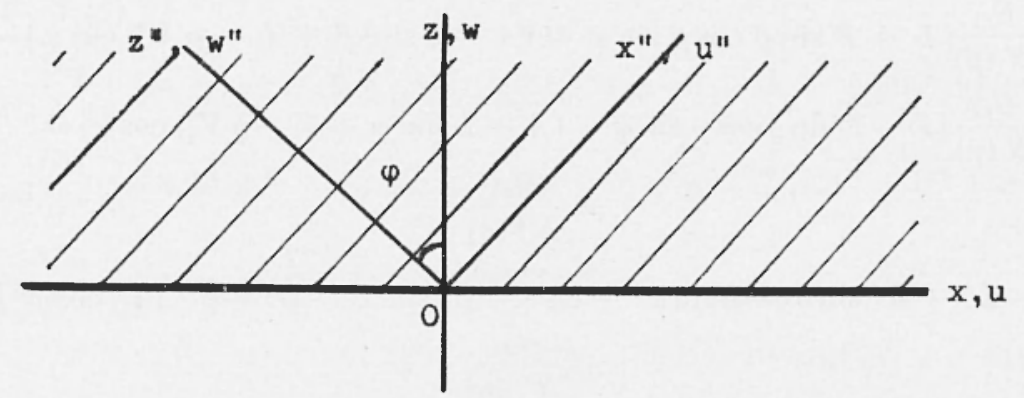

Fig. I - OZ, the normal to the boundry $z=0, O Z^{\prime \prime}$, the symmetry axis

$\begin{array}{cccc} & x & y & z \\ x^{\prime \prime} & \cos \varphi & 0 & -\sin \varphi \\ y^{\prime \prime} & 0 & 1 & 0 \\ z^{\prime \prime} & \sin \varphi & 0 & \cos \varphi\end{array}$

One hils the followings boundry conditions (the two media in welded contact):

$$
\begin{aligned}
u(x,+0, t) & =u(x,-0, t) \\
w(x,+0, t) & =v(x,-0, t) \\
\tau_{z x}(x,+0, t) & =\tau_{z x}(x,-0, t) \\
\tau_{z z}(x,+0, t) & =\tau_{z z}(x,-0, t) .
\end{aligned}
$$

With these conditions one gets:

$-\frac{G_{e}(L+E)}{N(\alpha)} \sin \alpha \cos \alpha \cos \varphi-\frac{G_{e}}{N(\alpha)}\left(\overline{A-L} \sin ^{2} \alpha+L-\varrho V^{2}\right) \sin \varphi+$ $+\frac{G_{r 1}}{N(\beta)}\left[-\left(L+\overline{H^{\prime}}\right) \sin \beta \cos \beta \cos \varphi+\left(\overline{A-L} \sin ^{2} \beta+L-\varrho V_{1}^{2}\right) \sin \varphi\right]+$ $+\frac{G_{r 2}}{N(\gamma)}\left[--(\overline{L+F}) \sin \gamma \cos \gamma \cos \varphi+\left(\overline{A-L} \sin ^{2} \gamma+L-\varrho V_{2}^{2}\right) \sin \varphi\right]=$ 


$$
-\frac{G_{d 1}}{N^{*}(\delta)}
$$

$\cdot\left[-\bar{L}^{*}+F^{*} \sin \delta \cos \delta \cos \varphi^{*}+\left(\overline{A^{*}-L^{*}} \sin ^{2} \delta+L^{*}-Q^{*} V_{1}^{*}\right) \sin \varphi^{*}\right]+$

$$
+\frac{G_{d 2}}{N^{*}(\eta)}
$$

. $\left[\overline{L^{*}+I^{*}} \sin \eta \cos \eta \cos \varphi^{*}+\left(\overline{A^{*}-L^{*}} \sin ^{2} \eta+L^{*}-\varrho^{*} V_{2}^{* 2}\right) \sin \varphi^{*}\right]$,

$\frac{G_{e}}{N(\alpha)}\left[-L+F \sin \alpha \cos \alpha \sin \varphi+\left(\overline{A-L} \sin ^{2} \alpha+L-\varrho V^{2}\right) \cos \varphi\right]-$ $-\frac{G_{r 1}}{N(\beta)}\left[\overline{L+F} \sin \beta \cos \beta \sin \varphi+\left(\overline{A-L} \sin ^{2} \beta+L-\varrho \overline{v_{1}}\right) \cos \varphi\right]-$ $=\frac{G_{r::}^{\prime}}{N(\gamma)}\left[\overline{L+F} \sin \varrho \cos \gamma \sin \varphi+\left(\overline{A-L} \sin ^{2} \gamma+L-\varrho V_{2}^{2}\right) \cos \varphi\right]=$

$$
=\frac{G_{d 1}}{N^{*}(\delta)} \text {. }
$$

$\left.\cdot\left[-\overline{L^{*}+F^{*}} \sin \delta \cos \delta \sin \varphi^{*}+\overline{\left(A^{*}-L^{*}\right.} \sin ^{2} \delta+L^{*}-\varrho^{*} V_{1}^{* 2}\right) \cos \varphi^{*}\right]+$

$$
+\frac{G_{d 2}}{N^{*}(\eta)}
$$

$\cdot\left[\overline{L^{*}+F^{*}} \sin \eta \cos \eta \sin \varphi^{*}-\left(\overline{A^{*}-L^{*}} \sin ^{2} \eta+L^{2}-\varrho^{*} V_{2}^{* 2}\right) \cos \varphi^{*}\right]$,

$$
-\frac{G_{e}}{V N(\alpha)} .
$$

. $\left\{\left[(\overline{A-F} \cdot \overline{L+F}+\overline{C-F} \cdot \overline{A-L}) \sin ^{2} \alpha+C\left(L-\varrho V^{2}\right)\right]\right.$.

$\left.\cdot \cos \alpha \sin \varphi \cos \varphi-L \sin \alpha \cos 2 \varphi\left(F+\varrho V^{2}-\overline{A+F} \sin ^{2} \alpha\right)\right\}+$

$+\frac{G_{r 1}}{N}(\beta) V_{1}\left\{\left[(\overline{A-F} \cdot \overline{L+H}+\overline{C-F} \cdot \overline{A-L}) \sin ^{2} \beta+\right.\right.$

$\left.\left.+C\left(L-\varrho V_{1}^{2}\right)\right](-\cos \beta \sin \varphi \cos \varphi)+L \sin \beta \cos 2 \varphi\left(F+\varrho V_{1}^{2}-\overline{A+F} \sin ^{2} \beta\right)\right\}+$

$+\frac{G_{r 2}}{N(\gamma) V_{2}}\left\{\left[(\overline{A-F} \cdot \overline{L+F}+\overline{C-F} \cdot \overline{A-L}) \sin ^{2} \gamma+\right.\right.$

$\left.\left.+C\left(\overline{L-\varrho V_{2}^{2}}\right)\right](-\cos \gamma \sin \varphi \cos \varphi)+L \sin \gamma \cos 2 \varphi\left(F+\varrho V_{2}^{2}-\overline{A+F} \sin ^{2} \gamma\right)\right\}=$

$=\frac{-G_{d 1}^{\prime}}{N^{*}(\delta) V_{1}^{*}}\left\{\left[\left(\overline{A^{*}-F^{*}} \cdot \overline{L^{*}+F^{*}}+\overline{C^{*}-F^{*}} \cdot \overline{A^{*}-L^{*}}\right) \sin ^{2} \delta+\right.\right.$

$\left.+C^{* *}\left(\overline{L^{*}-Q^{*} V_{1}^{* 2}}\right)\right] \sin \varphi^{*} \cos \varphi^{*} \cos \delta+L^{*} \sin \delta \cos 2 \varphi^{*}$.

$\left.\cdot\left(F^{*}+\varrho^{*} V_{1}^{* 2}-\overline{A^{*}+F^{*}} \sin ^{2} \delta\right)\right\}+$ 
$+\frac{G_{d 2}}{N^{*}(\eta) V_{2}^{;}}\left\{\left[\left(\overline{A^{*}-F^{*}} \cdot \overline{L^{*}+F^{*}}+\overline{C^{*}-F^{*}} \cdot \overline{A^{*}-L^{*}}\right) \sin ^{2} \eta+\right.\right.$ $\left.+C^{*} \cdot\left(\overline{L^{*}-\varrho^{*} V_{2}^{* 2}}\right)\right] \sin \varphi^{*} \cos \varphi^{*} \cos \eta+L^{*} \sin \eta \cos 2 \varphi^{*}\left(F^{*}+\right.$ $\left.\left.+\varrho^{*} \Gamma_{2}^{* 2}-\overline{A^{*}+F^{* *}} \sin ^{2} \eta\right)\right\}$,

$\frac{G_{e}}{V N(\alpha)}\left[h_{e}(\alpha, \varphi)+L \sin \alpha \sin 2 \varphi\left(\mathrm{F}+\varrho \Gamma^{2}-\overline{A+F} \sin ^{2} \alpha\right)\right]+$ $+\frac{G}{V_{1} Y(\beta)}\left[h_{1}(\beta, \varphi)+L \sin \beta \sin 2 \varphi\left(\mathrm{F}+\varrho V_{1}^{2}-\overline{A+F} \sin ^{2} \beta\right)\right]+$ $+\frac{G_{r 2}^{r}}{V_{2}} \frac{V^{r}}{(\gamma)}\left[h_{2}(\gamma, \varphi)+L \sin \gamma \sin 2 \varphi\left(F+\varrho V_{2}^{2}-\overline{A+F} \sin ^{2} \gamma\right)\right]=$ $=\frac{\tilde{u}_{d 1}}{V_{1}^{*} N^{*}(\delta)}\left[h_{3}\left(\delta, \varphi^{*}\right)+L^{*} \sin \delta \sin 2 \varphi^{*}\left(F^{*}+\varrho^{*} V_{1}^{* 2}-\overline{A^{*}+F^{*}} \sin ^{2} \delta\right)\right]$ $-\frac{G_{d 2}}{\Gamma_{2}^{*} N^{*}(\eta)}\left[h_{4}\left(\eta, \varphi^{*}\right)+L^{*} \sin \eta \sin 2 \varphi^{*}\right.$.

$$
\left.\cdot\left(F^{*}+\varrho^{*} V_{2}^{* 2}-\overline{A^{*}+F^{*}} \sin ^{2} \eta\right)\right],
$$

with

$$
\begin{gathered}
\left.\quad h_{i}(\omega, \varphi)=\overline{(A-F} \cdot \overline{L+F}+\overline{A-L} \cdot \overline{C-F}\right) \sin ^{2} \omega \sin ^{2} \varphi+ \\
+\overline{F(L+F)-C(A-L)} \sin ^{2} \omega-\left(L-\varrho V_{i}^{2}\right) \overline{(F-C)} \sin ^{2} \varphi-C\left(L-\varrho V_{i}^{2}\right) .
\end{gathered}
$$

The index $i$ gives the two types of waves.

The solution of the Eqs. [16] gives the ratios of the amplitudes of the different derived waves to that of the incident wave.

(One must bear in mind that all the angles in Eqs. [16] are to be measured from the symmetry axes).

\section{A. - Spectal cases.}

A very common case arises when the symmetry axes in both media coincide with the normals to the boundary surface, for example in the crust, where the anisotropy is due to the layering. One can obtain the formulas for this case, when one puts $\varphi=\varphi^{*}=0$ in the above Eqs. [16]. So one gets:

$$
a_{1 k} \cdot G_{k}=a_{10} \cdot G_{e} \quad(i=1, \ldots, 4)
$$


with

$$
G_{1}=G_{r 1}, \quad G_{2}-G_{+2}, \quad G_{3}=G_{d 1}, \quad G_{4}=G_{d 2}
$$

and

$$
\begin{aligned}
& a_{11}=-(L+F) \sin \beta \cos \beta / N(\beta) \\
& a_{12}=-(L+F) \sin \gamma \cos \gamma / N(\gamma) \\
& a_{13}=+\left(L^{*}+F^{*}\right) \sin \delta \cos \delta / N^{*}(\delta) \\
& a_{14}=-\left(L^{*}+F^{*}\right) \sin \eta \cos \eta / N^{*}(\eta) \\
& a_{10}=+(L+F) \sin \alpha \cos \alpha / N(\alpha) \\
& a_{21}=-\left(\overline{A-L} \sin ^{2} \beta+L-\varrho V_{1}^{2}\right) / N(\beta) \\
& a_{22}=-\left(\overline{A-L} \sin ^{2} \gamma+L-\varrho V_{2}^{2}\right) / N(\gamma) \\
& a_{23}=-\left(\overline{A^{*}--} L^{*} \sin ^{2} \delta+L^{*}-\varrho^{*} \mathrm{~V}_{\mathrm{I}}^{*}\right) / N^{*}(\delta) \\
& a_{24}=+\left(\overline{A^{*}}-L^{*} \sin ^{2} \eta+L^{*}-Q^{*} V_{2}^{* 2}\right) / N^{*}(\eta) \\
& a_{20}=-\left(\overline{A-L} \sin ^{2} \alpha+L-\varrho V^{2}\right) / N(\alpha) \\
& \left.a_{31}=-L \sin \beta \overline{(A+F} \sin ^{2} \beta-F-\varrho V_{1}^{2}\right) / N(\beta) V_{1} \\
& \left.a_{32}=-L \sin \gamma \overline{(A+F} \sin ^{2} \gamma-F-\varrho V_{i}^{2}\right) / N(\gamma) V_{2} \\
& \left.a_{33}=-L^{*} \sin \delta \overline{\left(A^{*}+F^{*}\right.} \sin ^{2} \delta-F^{*}-\varrho^{*} V_{1}^{*}\right) / N^{*}(\delta) V_{1}^{*} \\
& \left.a_{34}=+L^{*} \sin \eta \overline{\left(A^{*}+F^{*}\right.} \sin ^{2} \eta-F^{*}-Q^{*} V_{2}^{*}\right) / N^{*}(\eta) V_{2}^{*} \\
& \left.a_{30}=-L \sin \alpha \overline{(A+F} \sin ^{2} \alpha-F-\varrho V^{2}\right) / N(\alpha) V \\
& a_{41}=+\left[\overline{C(A-L)-F(L+F)} \sin ^{2} \beta+C\left(L-\varrho V_{1}^{2}\right)\right] \cos \beta / N(\beta) V_{1} \\
& \left.a_{42}=+[\overline{C(A-L)-F(L+F}) \sin ^{2} \gamma+C\left(L-\varrho V_{2}^{2}\right)\right] \cos \gamma / N(\gamma) V_{2} \\
& a_{43}=-\left[\overline{C^{*}\left(A^{*}-L^{*}\right)-F^{*}\left(L^{*}+F^{*}\right)} \sin ^{2} \delta+C^{*}\left(L^{*}-Q^{*} V_{1}^{* 2}\right)\right] \\
& \cos \delta / N^{*}(\delta) V_{1}^{*} \\
& \left.a_{44}=+\left[\overline{C^{*}\left(A^{*}-L^{*}\right)-F^{*}\left(L^{*}+F^{*}\right.}\right) \sin ^{2} \eta+C^{*}\left(L^{*}-\varrho^{*} V_{2}^{*}\right)\right] \\
& \cos \eta / N^{*}(\eta) V_{2}^{*} \\
& \left.a_{40}=-[\overline{C(A-L)-F(L+F}) \sin ^{2} \alpha+C\left(L-\varrho V^{2}\right)\right] \cos \alpha / N(\alpha) V \text {. }
\end{aligned}
$$

The different angles (see Fig. 2) are to be determined from the Snell's law, wheih holds for the wave normals (and not for the rays) Eq. [12].

With

$$
\frac{\sin \alpha}{V(\alpha)}=n
$$


one obtains:

$\sin \beta(\nu)=n ! \frac{\overline{\left(a+b n^{2}\right) \pm \sqrt{\left(a+b n^{2}\right)^{2}-e\left(\varrho^{2}-f n^{2}+g n^{4}\right)}}}{2\left(\varrho^{2}-f n^{2}+g^{1} n^{4}\right)}$

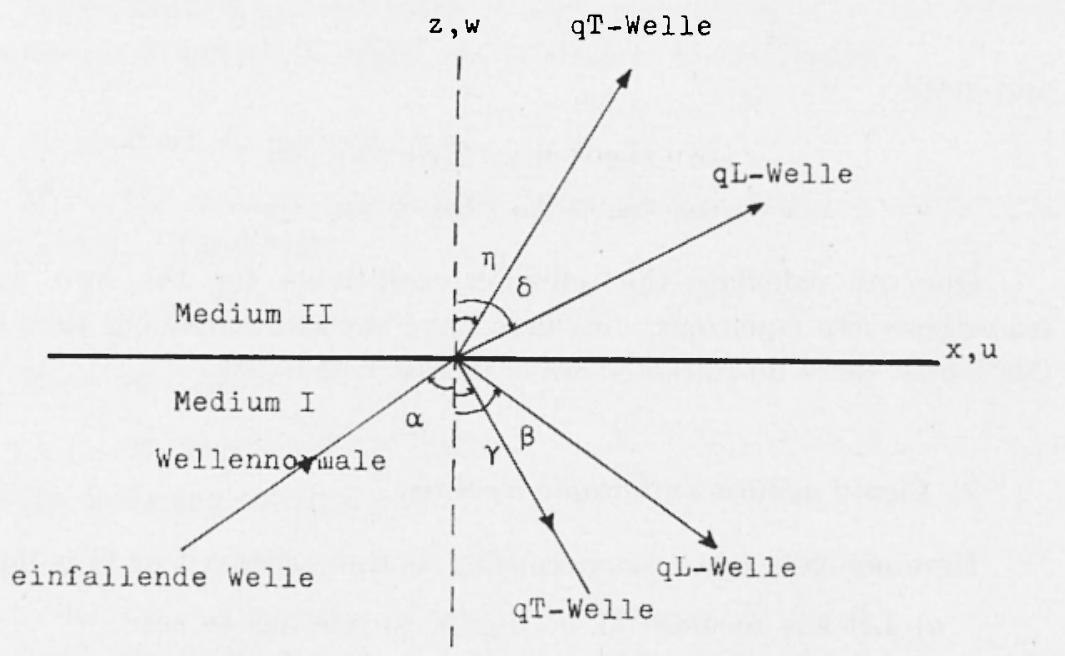

Fig. 2 - Angles, at which the different waves are reflected and refracted. Wellennormale $=$ wave normal, einfallende Welle $=$ incident wave, $q L$ and $q T$-Welle $=q L$ - (I type) and $q T$ - (II type) wave.

with

$$
\begin{aligned}
a & =\varrho(L+C), \quad e=4 L C, \quad f-\varrho(A-C) \\
b & =(L+F)^{2}-C(A-L)+L(C-L), \\
g & =(L+F)^{2}-(A-L)(C-L) .
\end{aligned}
$$

where, as before, the plus sign belongs to the wave of I type and to the angle $\beta$. A similar expression holds for the angles in medium II.

\section{B. - Further Spectal cases.}

Uptil now it was assumed that both of the media were transversal isotrope. In the natural conditions one finds very often that one of them is very approximately isotrope, e.g. if the strata underlying the oceans were anisotrope $\left({ }^{22}\right)$. In the following are given some few of such interesting cases. 
1) Free Surface (surface of the Earth for example).

This means that the second medium is practically vacuum. In other words the elastic constants as well as the density vanishes there. In Eq. [17] one sets therefore:

$$
A^{*}=C^{*}=L^{*}=F^{*}=A^{*}=0
$$

and gets:

$$
\begin{aligned}
& a_{31} \cdot G_{r 1}+a_{32} \cdot G_{r 2}=a_{30} \cdot G_{e} \\
& a_{41} \cdot G_{r 1}+a_{42} \cdot G_{r 2}=a_{40} \cdot G_{e} .
\end{aligned}
$$

One can calculate the reflexion coefficients for the two waves from these two equations. $a_{i k}$ here have the same meaning as in [17]. Obviously there no refracted wave in this case exists.

\section{2) Liquid against anisotropic medium.}

Here one gets two cases according as the medium $I$ or II is liquid:

a) Let the medium II be liquid, so one has to set:

$$
\begin{aligned}
& A^{*}=C^{*}=F^{*}=\lambda^{*} \\
& L^{*}=O
\end{aligned}
$$

(This is valid for isotropic liquids; they can always be considered to be isotropic).

As the liquids can glide against solid, the continuity of the tangential displacements cannot be assumed to hold; one can neglect it. With the above mentioned changes in the elastic constants the following $a_{i k}$ are simplified to:

$$
\begin{aligned}
& a_{11}=a_{12}=a_{13}=a_{14}=a_{24}=a_{34}=a_{44}=a_{33}=\hat{\mathrm{v}}, \\
& a_{23}=+\cos \delta, \\
& a_{43}=Q^{*} V_{1}^{*} .
\end{aligned}
$$

With these changes in Eq. [17] one can calculate the reflexion and trunsmission coefficients.

b) Let now the medium I be the liquid. One has then to set:

$$
\begin{aligned}
& A=C=F=\lambda, \\
& L=O
\end{aligned}
$$

and 
and get:

$$
\begin{aligned}
& a_{11}=a_{12}-a_{13}=a_{14}=a_{22}=a_{31}=a_{32}=a_{42}=0, \\
& a_{20}=+\cos \alpha \quad a_{30}=0 \quad a_{40}=\varrho V \\
& a_{21}=+\cos \beta \quad a_{41}=-\varrho V .
\end{aligned}
$$

In the present case the incident wave can only be of $P$-type (type I) because no $S$-waves (II type) can propagate in the liquids.

3) Medium I, isotropic solid.

Here the source can radiate pure longitudinal or transversal waves only. Therefore:

a) incident $P$-wave

Here one has:

$$
A=C=\lambda+2 \mu, \quad L=\mu, \quad H^{\top}=\lambda,
$$

and the following changes in $a_{\imath_{k}}$ :

$$
\begin{array}{ll}
a_{11}=-\sin \beta, & a_{12}=-\cos \gamma, \\
a_{10}=+\sin \alpha, & a_{21}=+\cos \beta, \\
a_{22}=-\sin \gamma, & a_{20}=+\cos \alpha, \\
a_{31}=+\left(\varrho V_{2}^{2} / V_{1}\right) \cdot \sin 2 \beta, & \\
a_{32}=\varrho V_{2} \cos 2 \gamma, & a_{41}=-\varrho V_{1} \cos 2 \beta, \\
a_{30}=+\left(\varrho V_{2}^{2} / V_{1}\right) \cdot \sin 2 \alpha, & \\
a_{42}=+\varrho V_{2} \sin 2 \gamma, & a_{40}=+\varrho V_{1} \cos 2 a . \\
\text { b) incident S-wave } &
\end{array}
$$

Here one has similarly:

$$
\begin{aligned}
& a_{10}=+\cos \alpha, \\
& a_{20}=-\sin \alpha, \\
& a_{30}=+\varrho \bar{\gamma}_{2} \cos 2 \alpha, \\
& a_{40}=-\varrho V_{2} \sin 2 \alpha .
\end{aligned}
$$

All other $a_{i k}$ remain as in [3a].

In the above expressions $V_{1}\left(V_{2}\right)$ is the velocity of $P(S)$ waves in the isotropic medium.

4) Medium II, isotropic solid.

The source can send here the $q L$ - or $q T$-wavess. Both of the types can be treated here as before together because of the similar almost identical (except in $V$ ), form of the two expressions. 
The transformation to isotropy in the medium II can be performed as follows:

$$
L^{*}=\mu^{*}, A^{*}=C^{*}=\lambda^{*}+2 \mu^{*}, F^{*}=\lambda^{*} .
$$

One gets then:

$$
\begin{aligned}
& a_{13}=+\cos \delta, \quad a_{23}=+\sin \delta, \\
& a_{33}=+\varrho^{*} V_{2}^{* 2} \sin 2 \delta / V^{*}, \quad a_{13}+\varrho^{*} V_{1}^{*} \cos 2 \delta \text {, } \\
& a_{14}=-\cos \eta, \quad a_{24}=+\sin \eta, \\
& a_{34}=-\varrho^{*} V_{2}^{*} \cos 2 \eta, \quad a_{44}=+\varrho^{*} V_{i}^{*} \sin 2 \eta \text {. }
\end{aligned}
$$

It can be shown very easily that Eq. [17] grive the same equations determining the amplitude ratios of the derived waves to that of the incident wave as in the case of isotropy, if one transforms the elastic constants of the anisotropic to those of the isotropic media (19) For example one gets the following equations for the incident $P$-wave:

$$
\begin{aligned}
& \begin{array}{ccccc}
G_{r 1} & G_{r 2} & G_{d 1} & G_{d 2} & G_{e}
\end{array} \\
& +\sin \beta+\cos \gamma \quad-\sin \delta \quad-\cos \eta=-\sin \beta \\
& -\cos \beta+\sin \gamma \quad-\cos \delta \quad-\sin \eta=-\cos \beta \\
& +\sin 2 \beta+\frac{V_{1}}{V_{2}} \cos 2 \gamma+\frac{\varrho^{*} V_{2}^{*} V_{1}}{\varrho V_{2} V_{1}^{*}} \cdot-\frac{\varrho_{*} V_{1} V_{2}^{*}}{\varrho V_{2} V_{2}}=+\sin 2 \beta \\
& \cdot \sin 2 \delta \quad \cdot \quad \cos 2 \eta \\
& -\cos 2 \beta+\frac{V_{2}}{V_{1}} \sin 2 \gamma+\frac{\varrho^{*} V_{2}^{*}}{\varrho V_{1}} \cos 2 \delta+\frac{\varrho^{*} V_{2}^{*}}{\varrho V_{1}} \sin 2 \eta=\cos 2 \gamma .
\end{aligned}
$$

\section{Results.}

In the following diagrams the calculated results for the reflexion and transmission coefficients as function of the angle of incidence are shown for the incident $q L$-wave (I type). The results were obtained with the help of the computer Telefunken $T R 4$ of the Bayerische Akademie der Wissen-Schaften. The coefficients have been calculated for every fifth degree, and for every degree near the critical angle. The direction of the particle motion was also tabulated, from the Eq. [9]. These 
tables are not reproduced here owing to their large volume. They can however be seen in the library of the Institut für Angewandte Geophysik der Universität München.

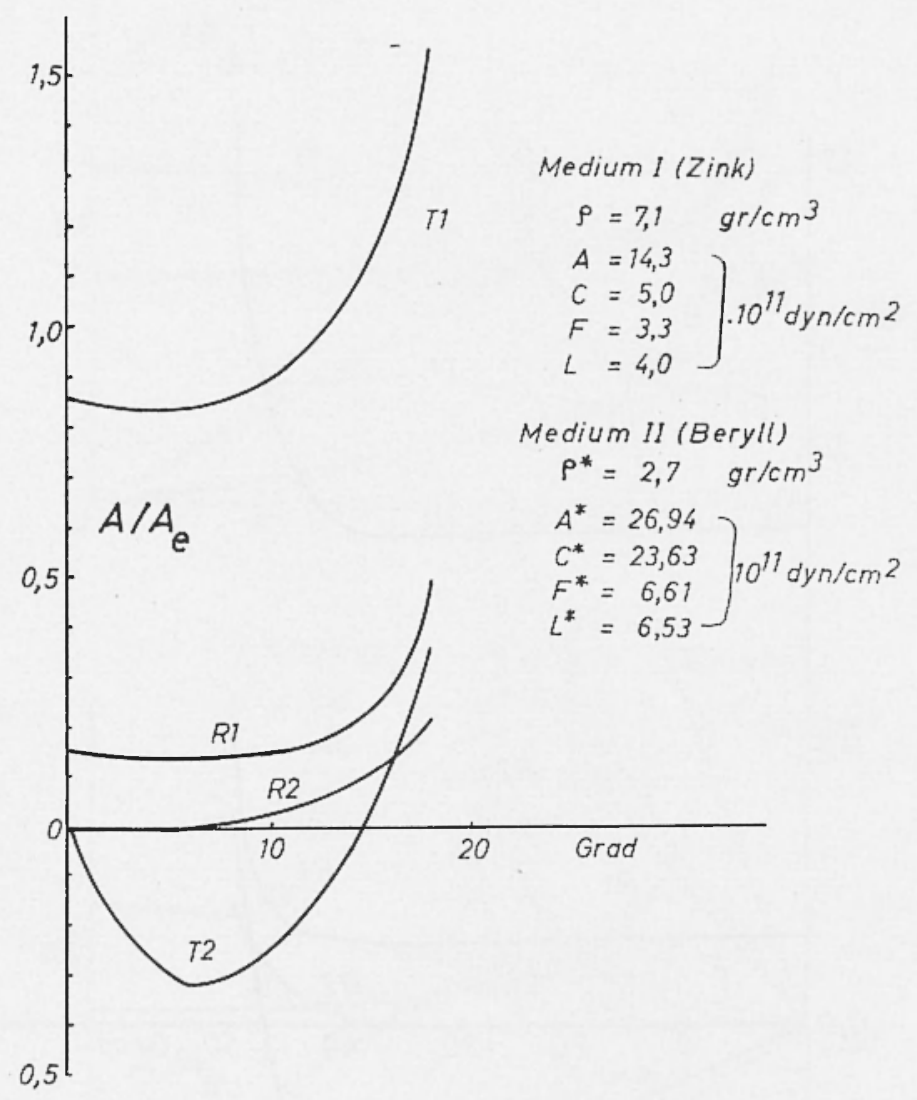

Fig. 3a: The amplitude ratios.

$R 1$ and $R 2$ : reflexion coefficients for waves of I type

$T 1$ and T2: transmission coefficients for waves of II type.

The angle of incidence has been taken $(a)$ as that of the wave normals $(b)$ as that of the ray. $R 1$ and $R 2$ are the reflexion coeffcients for the wave of the I type (quasilongitudinal) and II (quasitransversal) type respectively. $T 1$ and $T 2$ are similarly the transmission coefficients. 
(In the results presented here the normal to the discontinuity surface coincides with the symmetry axis in both media).

Fig. $3 a$ shows the amplitude ratios of the derived waves as a function of the direction of the wave normals for the media: zinc

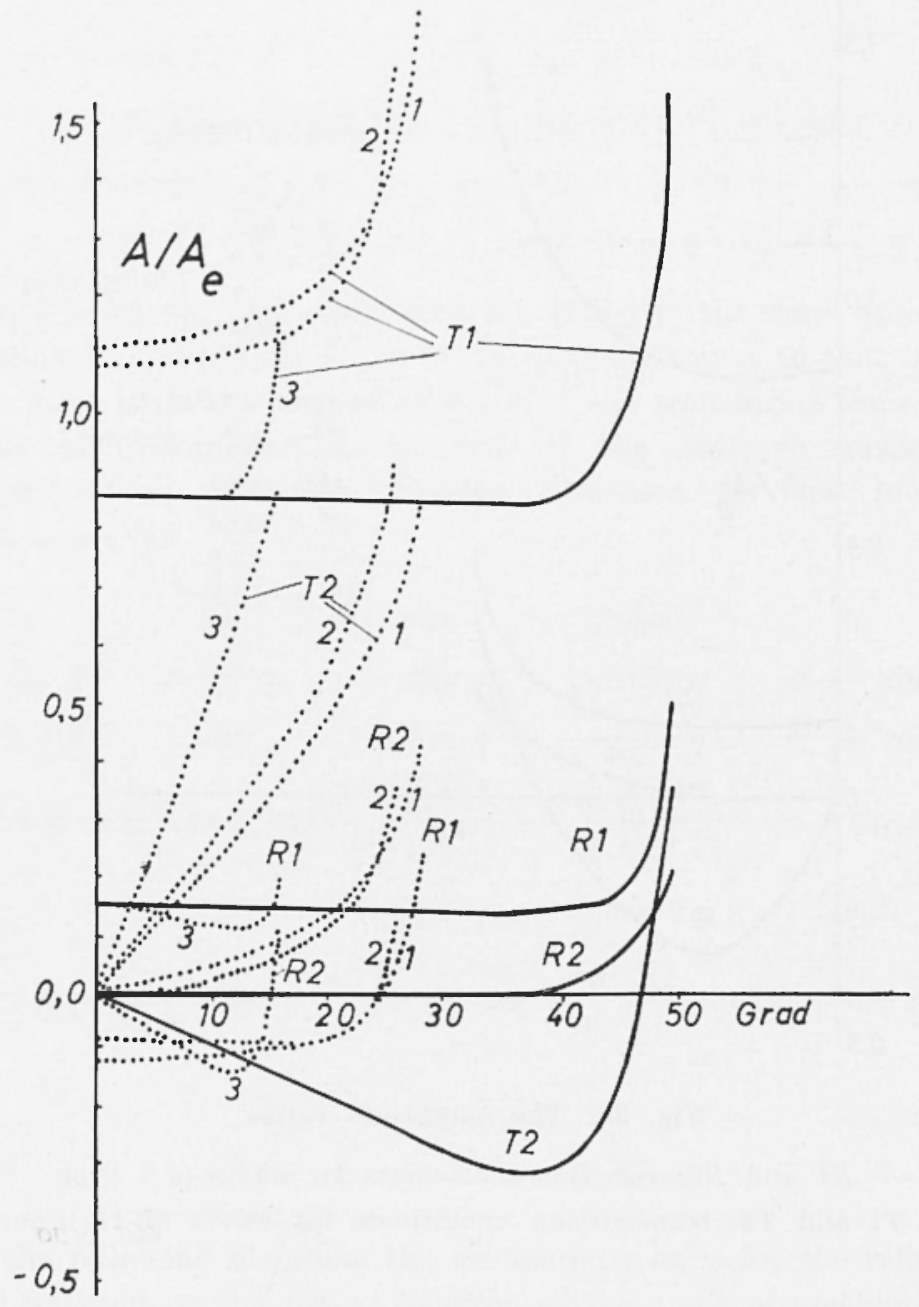

ANGLE OF INCIDENCE (RAYS)

Fig. $3 b$ - The amplitude ratios.

The full drawn curves are for zinc/beryl, the dotted for three hypothetical isotropic media, which have some of their elastic constants common with zinc and beryl. The numbers on the dotted curves point to these different cases, for the constants see text. 
against beryl. The elastic constants were taken from Hearmon (1961).
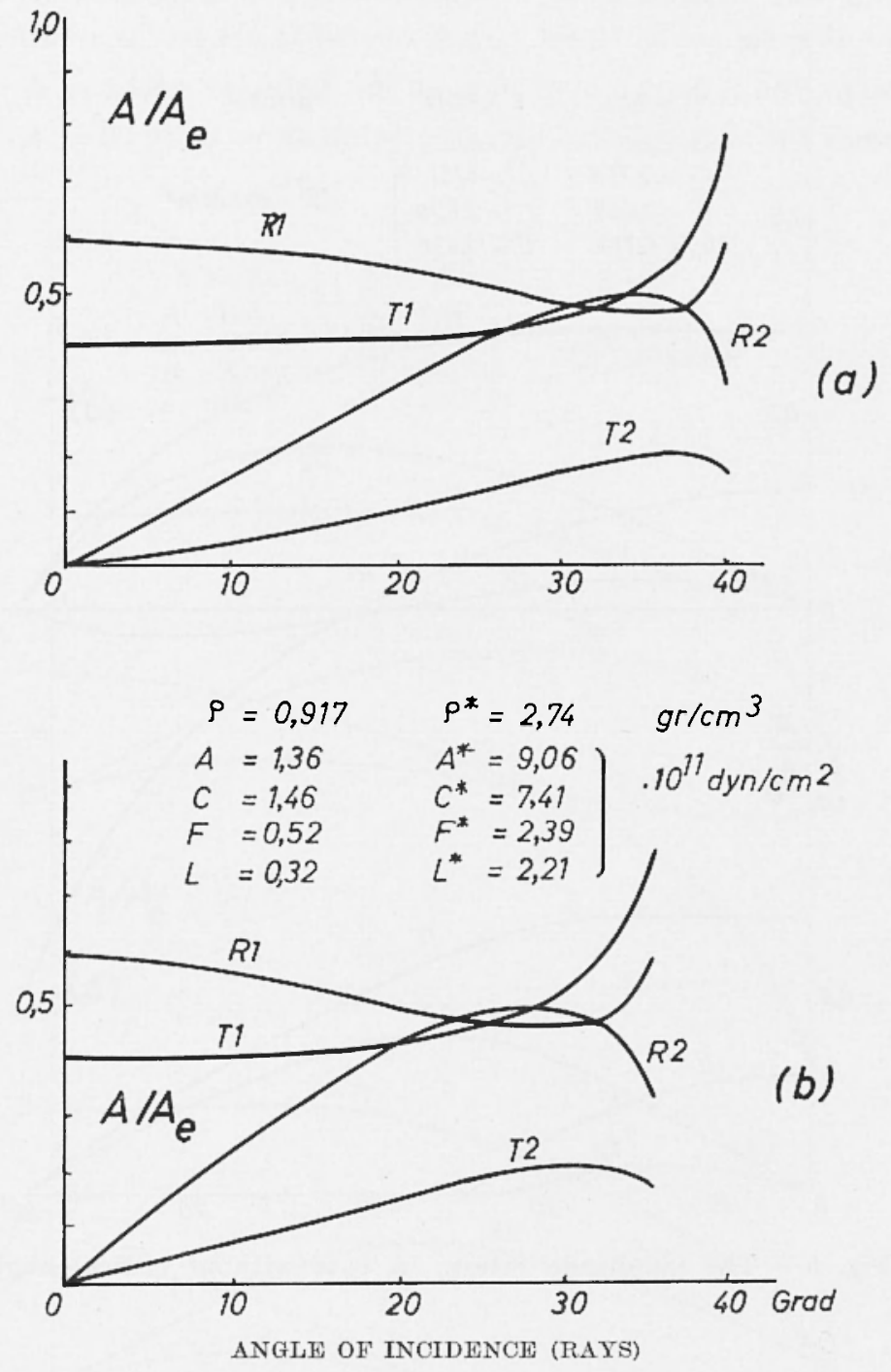

Fig. 4 - The amplitude ratios for ice/schists.

Fig. $3 b$ shows the same as function of the ray direction. In order to bring out clearly the difference between the coefficients for the isotropic and anisotropic media, the results are also shown in Fig. $3 b$ 
for three hypothetical isotropic media, which have some of the elastic constants common with those of the allied anisotropic media that is

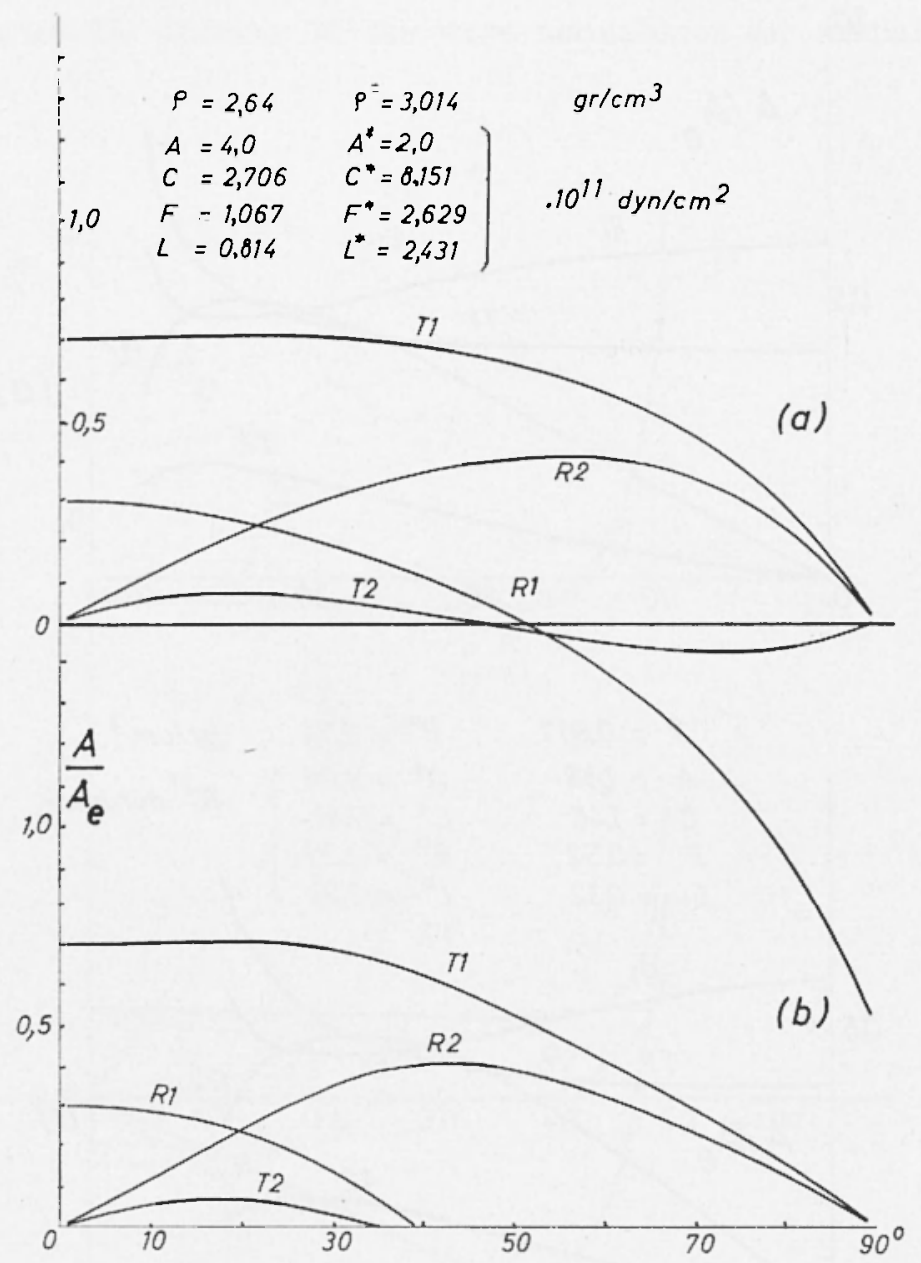

Fig. 5 - The amplitude ratios. A case without critical angle.

zine and beryl. These hypothetical media have the folloving constants:

$$
\begin{array}{ll}
\text { case 1: } & \varrho=7,1 \mathrm{~g} / \mathrm{cm}^{3} \quad(A=C=) \lambda+2 \mu=14,3 \cdot 10^{11}, \\
& (L=) \mu=4 \cdot 10^{11} \\
& \varrho^{*}=2,7 \mathrm{~g} / \mathrm{cm}^{3} \quad\left(A^{*}=C^{*}=\right) \lambda^{*}+2 \mu^{*}=23,63 \cdot 10^{11}, \\
& \left(L^{*}=\right) \mu^{*}=6,53 \cdot 10^{11}
\end{array}
$$


case 2: $(A=C=) \lambda+2 \mu=14,3 \cdot 10^{11} \quad(L=) \mu=5,5 \cdot 10^{11}$

$$
\left(A^{*}=C^{*}\right) \lambda^{*}+2 \mu^{*}=26,94 \cdot 10^{11}\left(L^{*}=\right) \mu^{*}=10,165 \cdot 10^{11}
$$

case 3: $(A=C=) \lambda+2 \mu=5 \cdot 10^{11}(L=) \mu=8,5 \cdot 10^{11}$

$$
\left(A^{*}=C^{*}=\right) \lambda^{*}+2 \mu^{*}=23,63 \cdot 10^{11}(L=) \mu^{*} 8,51 \cdot 10^{11}
$$

Zinc: $\quad A=14,3 \quad C=5,0 \quad F=3,3 \quad L=4,0$ (all in $10^{11}$ dyne $/ \mathrm{cm}^{2}$ )

Beryl $A=26,94 C=23,63 F^{\prime}=6,61 L=6,53$ (all in $10^{11}$ dyne $/ \mathrm{cm}^{2}$ )

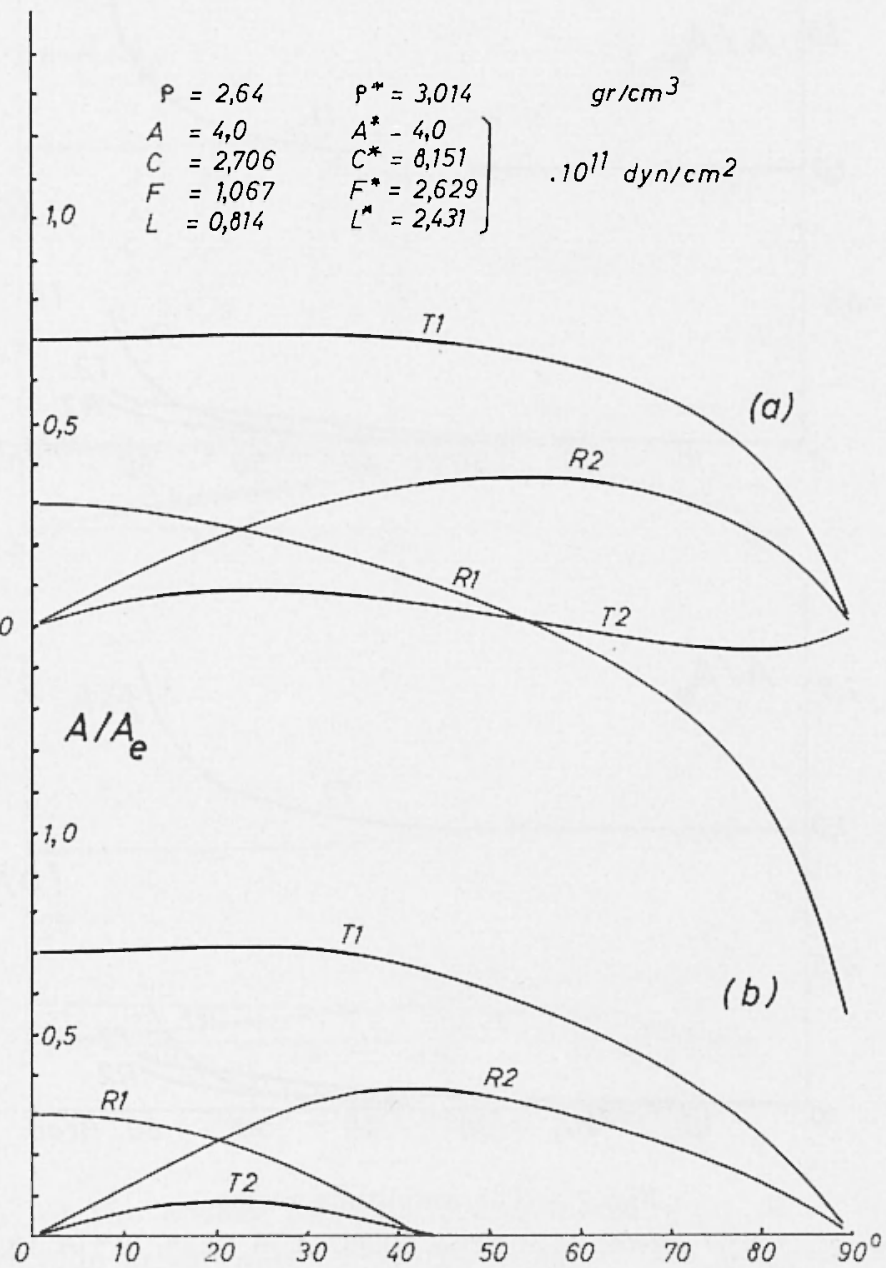

Fig. 6 - The amplitude ratios. Critical angle $90^{\circ}$.

(In the cases 2 and 3 as well as zine and beryl the densities are the same as in case 1 . The constants are in $d y n e / \mathrm{cm}^{2}$ ). 
The difference between the critical angles for the anisotropic and isotropic media is due to the fact that the ray directions deviate a lot from those of the wave normals in anisotropy, whereas in isotropy
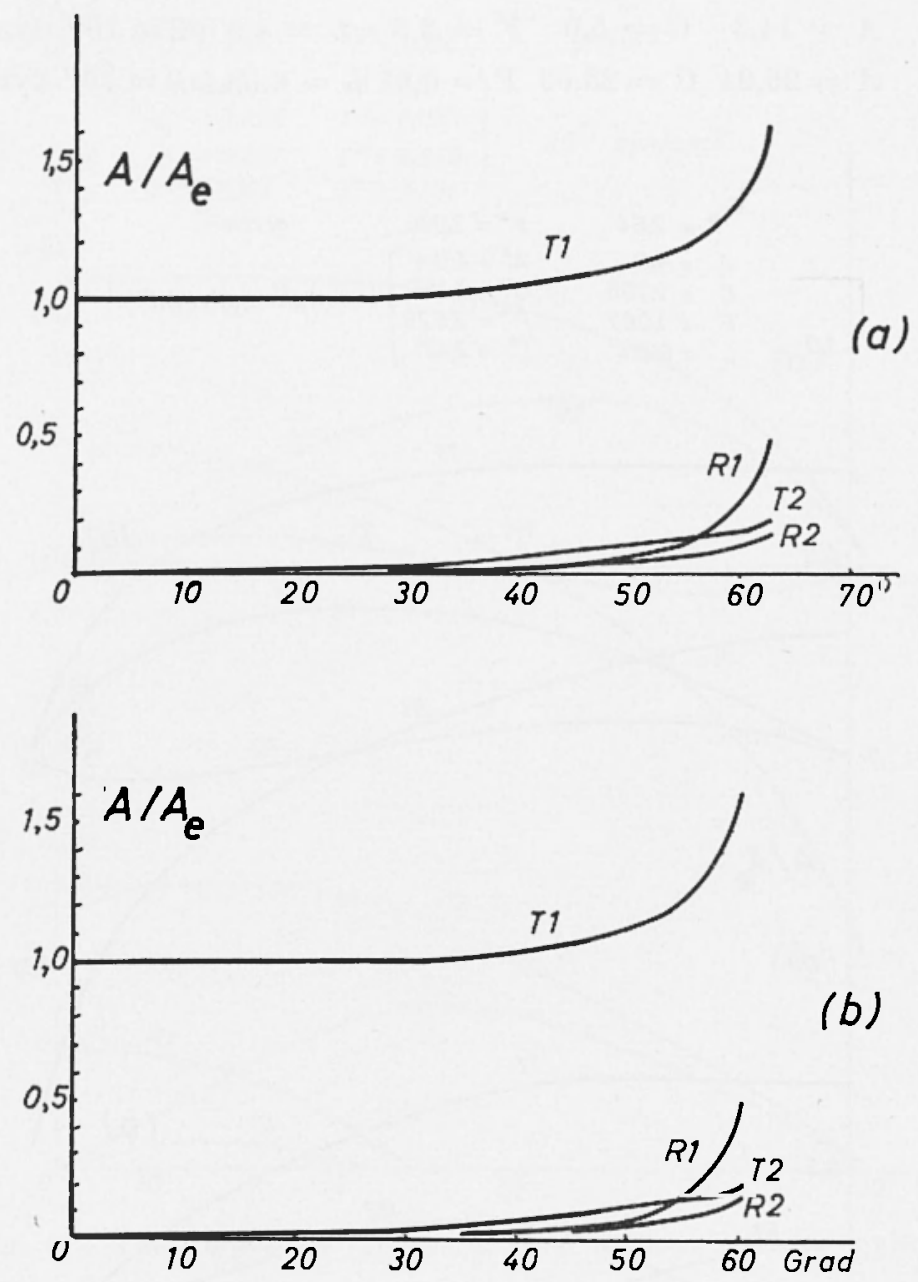

Fig. 7 - The amplitude ratios.

$$
\begin{gathered}
\varrho=\varrho^{*}=2,67 \mathrm{~g} / \mathrm{cm}^{3}, C=C^{*}=10,38, A=10,38, A^{*}=13,23, \\
F=F^{*}=4,41, L=L^{*}=2,98 \mathrm{in} 10^{11} \mathrm{dyne} / \mathrm{cm}^{2} .
\end{gathered}
$$

they are the same. As to the behaviour of the amplitude ratios the maximum deviation from the isotropic eases occurs for $7^{\prime 2}$. In the 
isotropic cases there is no extremum, while the anisotropy shows a minimum. Besides there is a phase difference of 180 degrees for the two media, for the whole range of angles except in the immediate neigh-
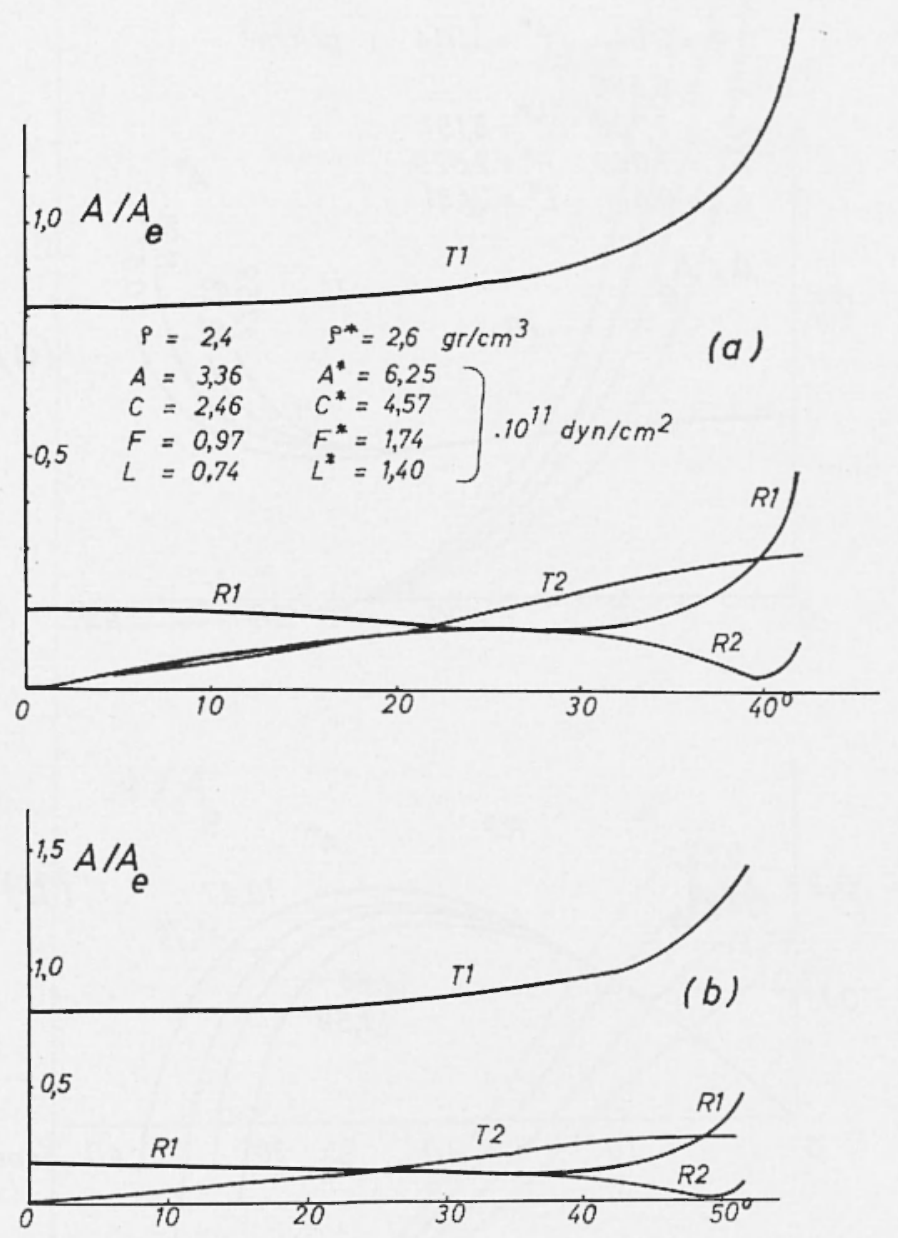

Fig. 8 - The amplitude ratios.

bourhood of the critical angles. The reflected wave of II type has less amplitude in the anisotropic media here. The reflected wave of the I type shows also a noticeable deviation. Apart from the difference in the critical angles, which exists for all the waves, the behaviour of the curves in both of the cases is similar excep for the phase difference of $180^{\circ}$ between them as with $T 2$. 
Fig. $4(a, b)$ shows the amplitude ratios as a function of the ray direction as well as wave normals. Here Medium $I$ is ice, while the medium II consists of the schistose rocks. (The elastic constants of
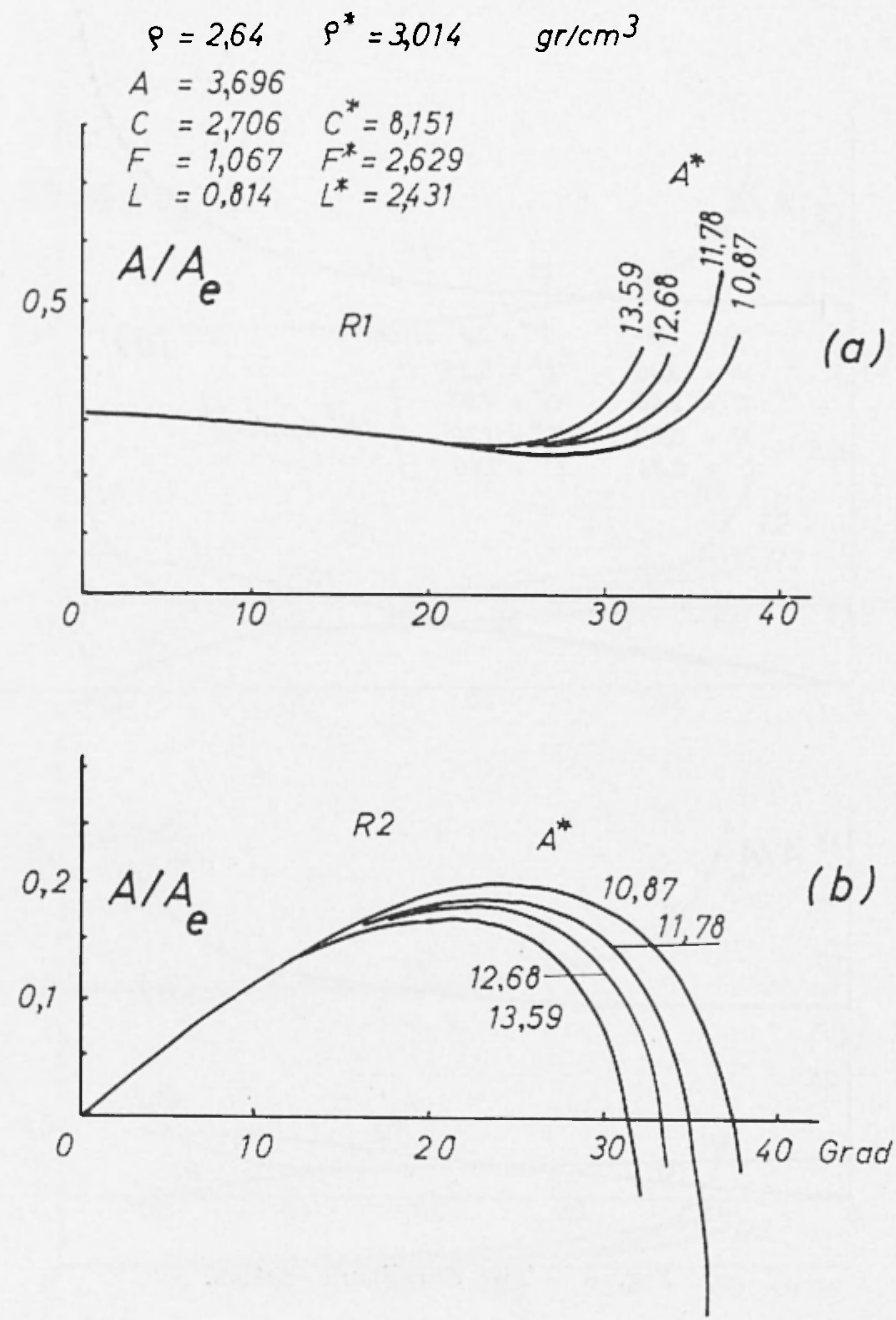

Fig. 9 - The influence of varying $A^{*}$ on the amplitudes of the reflected waves.

these schists were taken from Schmidt $\left({ }^{23}\right)$ ) The amplitudes of the reflected waves are greater than those of the refracted ones. It is to be noted that in the ice the velocity in the vertical direction is greater than that in the horizontal. 
REFLEXION AND REFRACTION COEFFICIENTS, ETC.

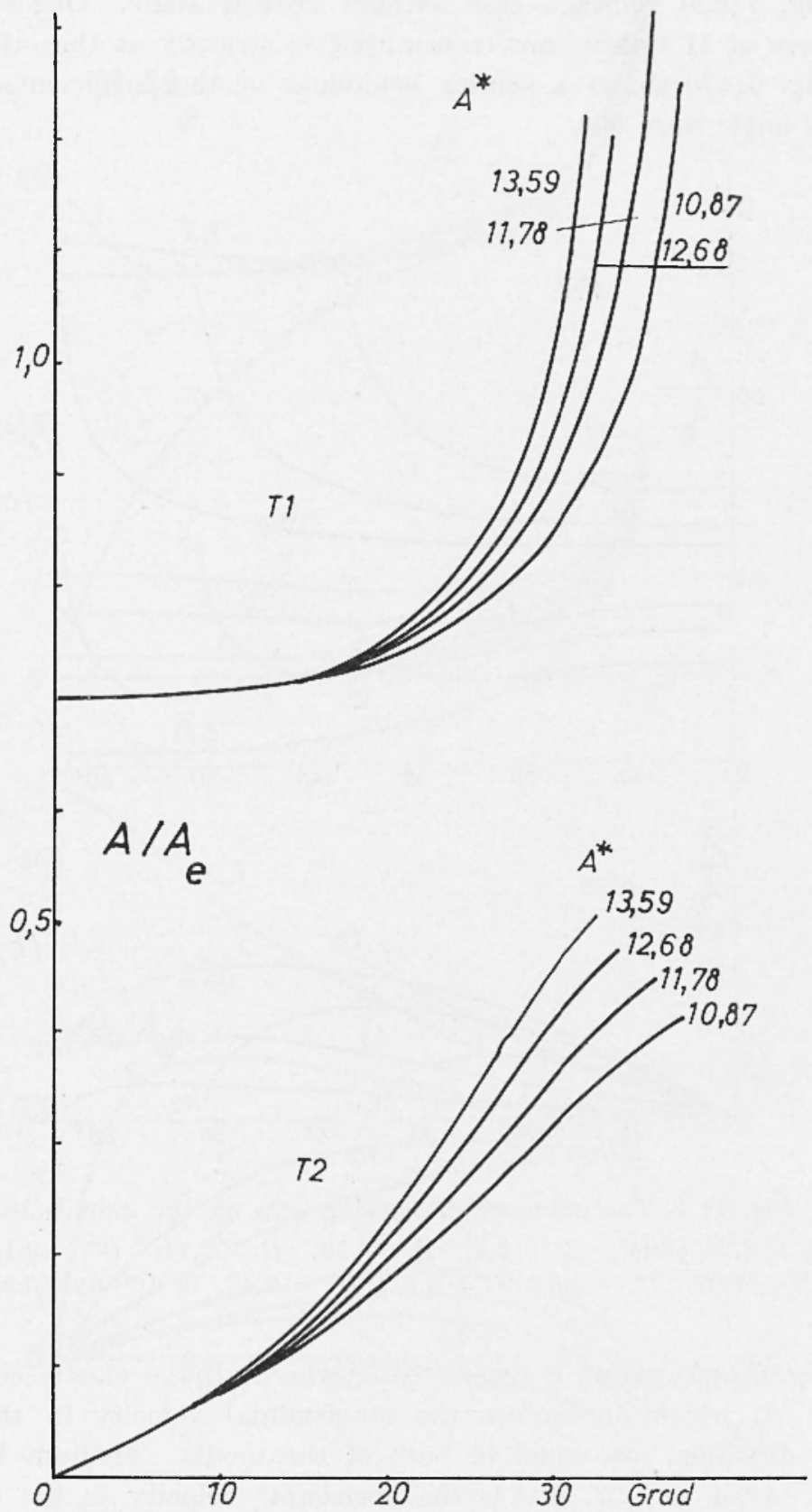

Fig. 10 - The influence of varying $A^{*}$ on the amplitudes of the refracted waves. 
Fig. $5(a, b)$ shows a case without critical angle. One sees that the wave of II type is not transmitted so strongly as the other one.

Fig. $6(a, b)$ shows a similar behaviour of the coefficients, if the critical angle were $90^{\circ}$.

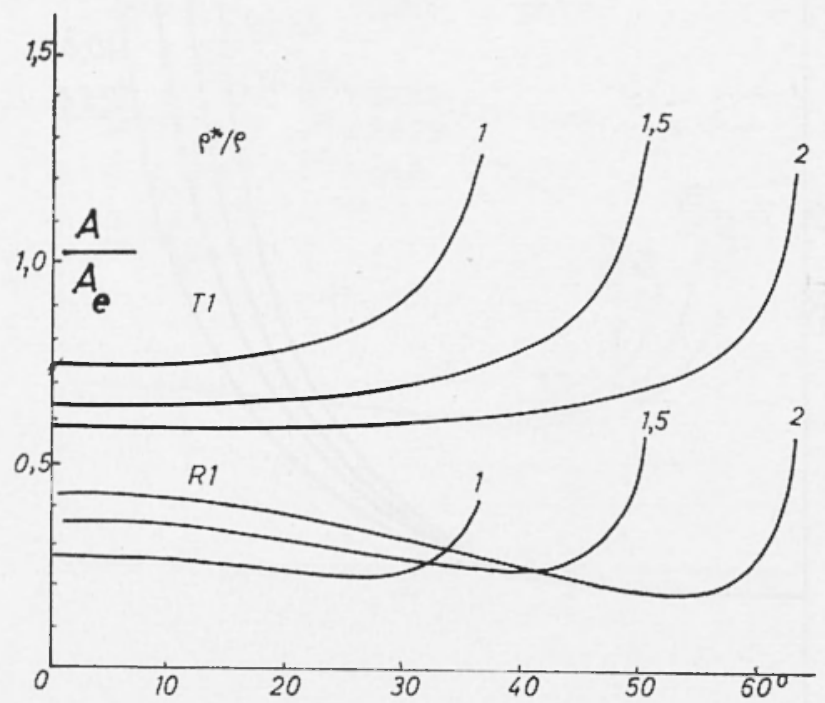

\section{(a)}

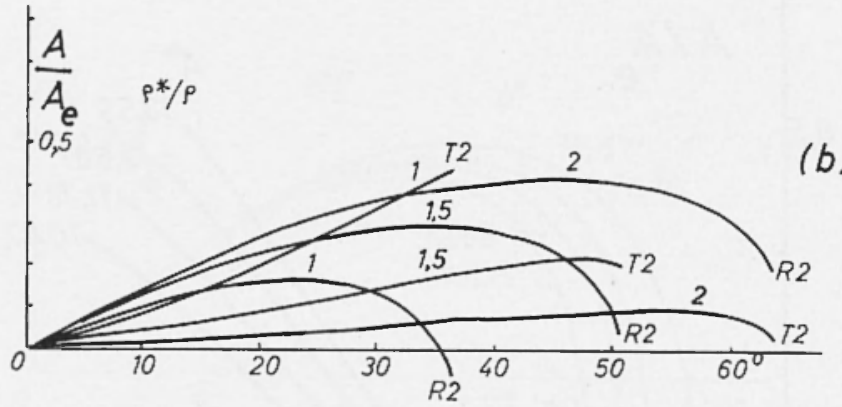

Fig. $11-$ The influence of varying $e^{*} / \varrho$ on the amplitude. $\varrho=2,64 \mathrm{~g} / \mathrm{cm}^{3}, \quad A=3,7, \quad A^{*}=10, \quad C=2,71, \quad C^{*}=8,15$, $F^{\prime}=1,07, F^{*}=2,63, L=0,81, L^{*}=2,43$, in $10^{11} \mathrm{dyne} / \mathrm{cm}^{2}$.

Fig. $7(a, b)$ shows a special case, where all the elastic constants except $A$, which determines the longitudinal velocity in the horizontal direction, are equal in both of the media. Medium $I$ is isotrope. $A^{*} / A=1,27$, that is the horizontal velocity in the medium II is about $13 \%$ higher than that in the first medium. Here the reflected waves (both types) have very small amplitudes up to about 
REFLEXION AND REFRACTION COEFFICIEATS, ETC.
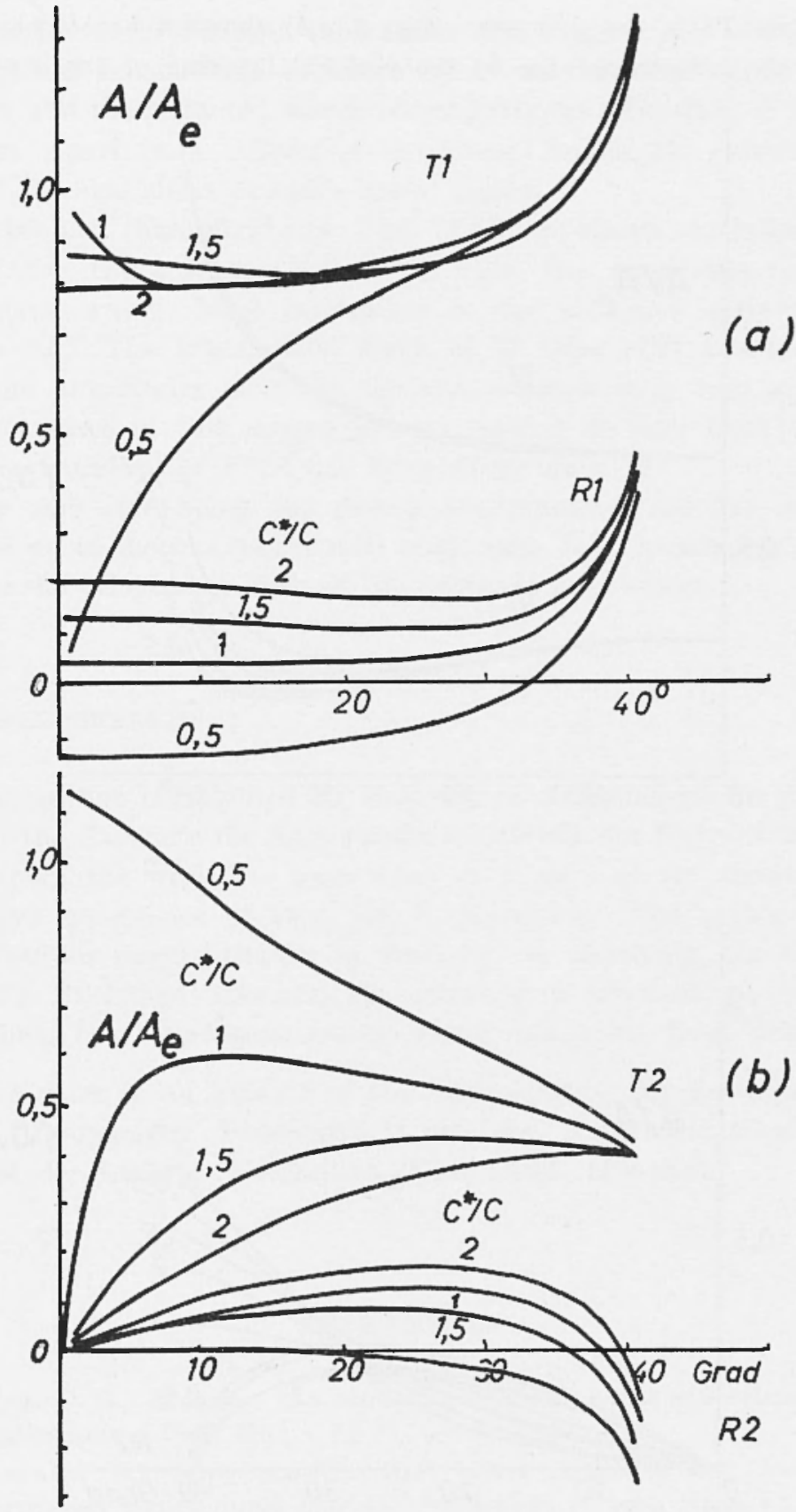

Fig. 12 - The influence of the variation of $C^{*} / C$ on the amplitudes (for the constants see Fig. 11). 
$50^{\circ}$ while $T 1$ is the greatest. Fig. $8(a, b)$ shows a case where both media are anisotropic due to the periodic layering of the sandstones
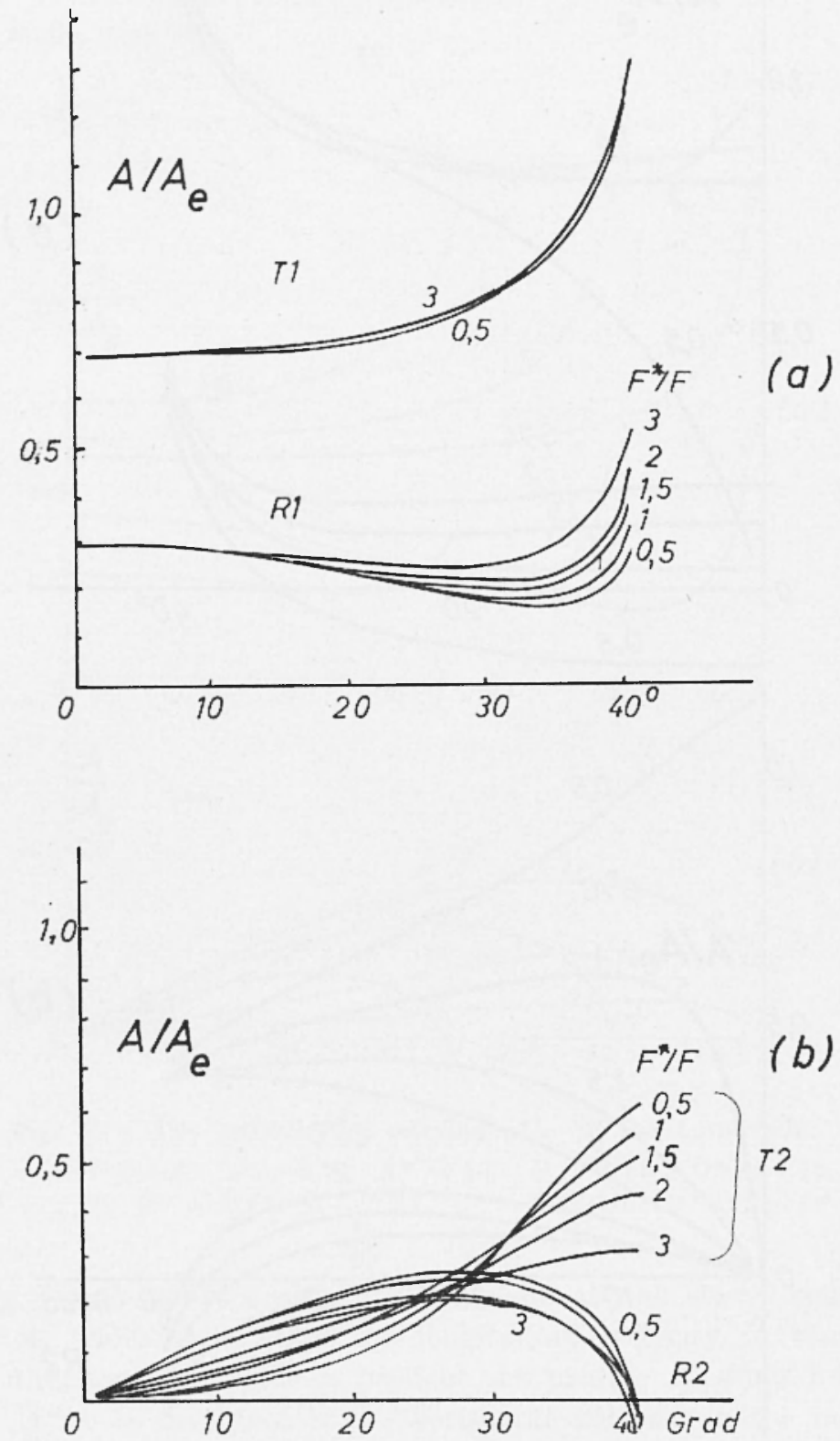

Fig. 13 - The influence of the variation of $F^{*} / F$ on the amplitudes (for the constants see Fig. 11). 
and limestones in different thichnesses $\left({ }^{13}\right)$. Figg. $9(a, b)$ and $10(a, b)$ show the influence of the variation of $A^{*}$ on the amplitudes of the reflected and the refracted waves respectively, as a function of the ray direction. Apart from shifting of the critical angles, the extremum of $R 1$ and $R 2$ also shifts towards lower angles.

In the last three diagrams (Figg. 11-13) are shown the influence of varying the ratios $\varrho^{*} / \varrho, C^{*} / C, F^{*} / F$ upon the amplitude ratios of the derived waves. Most interesting is the influence of $C^{*} / C$ for $C^{*} / C=0.5$. The transmitted wave of II type $(T 2)$ has here the maximum amplitudes near the vertical, whereas it is very small for the other cases in this range. It can further be seen from Fig. 13 that the variation of $F^{*} / F$ has little effect upon $T 1$.

For the time being the results are presented for the incident $q L$-wave up to the smallest critical angle only. It is intended to extend them to the other cases over all the angles in near future.

\section{ACKNOWLEDGEMENTS.}

The author is indebted to Prof. Dr. G. Angenheister for giving a place in the Institute für Angewandte Geophysik der Universität-München, where the work has been done as a part of Dr. thesis under the active supervision of Prof. Dr. E. Hardtwig. The author wishes to express his sincere thanks to Prof. Dr. F. Hardtwig, the German Student's Exchange (DAAD) for granting a scholarship, without whose help the calculation results would not have been realisable.

This work is an extract of the "Dissertation für Erlangung des Grades 'Doctor der Philosophie'" am der Naturwissenschaftlichen Fakultät der Lüdwig - Maximilian - Universität, München.

\section{REFERENCES}

(') Kмотт C. G., Reflexion and refraction of elastic waves with seismological applications. "Phil. Mag." 64-97, (1899).

(') Zoeppritz K., Erdbebenwellen Fll. Ueber Reflexion und Durchgang seismischer Wellen durch Unstetigleitsflaechen. "Goett. Nach.", (1919).

(3) Muskat M., Meres M. W., Reflexion and transmission coefficients for plane waves in elastic media. "Geop.", 115-48. (1940). 
(4) Konfoed O., Reflexion and transmission coefficients for plane longiludinal incident waves. "Geop. Pros. ", 304-51, (1962).

(5) Uirrig L. F., VaN Melde F. A., Velocity anisotropy in stratified media. "Geop. ", 774-9, (1955).

(') Негвig K., Elastische Wellen in anisotropen Medien. "Gerl. Beit. z. Geop. ", 177-211, (1958).

(7) Giesel W., Elastische Anisotropie in tecktonisch verformten Sedimentgesteinen. "Geop. Pros. ", 423 ff. (1963).

(8) STONeLey R., The seismological implications of aeolotropy in continental structure. "Mon. Not. Roy. Ast. Soc. Geop." Suppl. 343-53, (1949).

$\left.{ }^{(}\right)$STONeley R., Polarisation of the $S$ phase of seismograms. "Annali di Geofisica ", 3-8, (1951).

(10) Vvedenskaya A. V., Balakina L. M., Double refraction in the earth's mantle. "Bull. Izw. Acad. Sc. USSR ", (Geop) 814-9, (1959).

(11) Nuttli O., Whitsione J. D., On the determination of the polarisation of $S$ waves. "Bull. S. S. A.", 95-107, (1962).

(12) Meissner R., An interpretation of the wide angle measurements in the Bavarian Molasse. "Geop. Pros.", 7-16, (1966).

(13) Postma G. W., Wave propagation in stratified media. "Geop." 780806, (1955).

(14) BackUS G. E., Long wave elastic anisotropy produced by horizontal layering. "J. Geop. Res. ", 4427-40, (1962).

(15) OsSIpov 1. O., Reflexion and refraction of plane elastic waves at the boundry between two anisotropic media. "Bull. Izw. Acad. Sc. USSR" (Geop. Ser.), 424-31, (1961).

${ }^{(16)}$ Ossipov I. O., Refexion and refraction of elastic waves at the boundry of a liquid and solid anisotropic media. ibid. 12, (1961a).

(17) Musgrave M. J. P., Refexion and refraction of plane waves at a boundry between aeolotropic media. "Geop. J." " 406-18, (1960).

${ }^{(18)}$ Love A. E. H., A treatise on the mathematical theory of elasticity. "Camb. Uni. Press. ", (1927).

(19) BuUt 11., Ein Beitrag zur Theorie der Reflexion und Brechung elastischer Wellen an Unstetigkeitsflaechen. "Z. Geop.", 130-44, (1932).

(20) Gutenberg B., Theorie der Erdbeben. "Hb. Geop. ", IV, 42 ff. (1932).

(21) Ewing W. M., Jardetzky W. S., Press F., Elastic waves in layered media. "McGraw Hill. ", (1957).

(22) Hess H. H., Seismic anisotropy of the upper-most mantle, under oceans. "Nature " 203, ,4945, 629-31, (1964).

${ }^{(23)}$ Schmidt E., A case of anisotropy of seismic velocity. "Geoexpl. ", 28-35, (1964). 